\title{
Dynamic Modeling of Compliant Constant-Force Compression Mechanisms
}

\author{
Cameron Boyle \\ Larry L. Howell \\ Ihowell@byu.edu \\ Spencer P. Magleby \\ magleby@byu.edu \\ Mark S. Evans
}

Follow this and additional works at: https://scholarsarchive.byu.edu/facpub

Part of the Mechanical Engineering Commons

\section{Original Publication Citation}

Boyle, C., Howell, L.L., Magleby, S.P., and Evans, M.S., "Dynamic Modeling of Compliant ConstantForce Compression Mechanisms," Mechanism and Machine Theory, Vol. 38, No. 12, pp.

1469-1487, 23.

\section{BYU ScholarsArchive Citation}

Boyle, Cameron; Howell, Larry L.; Magleby, Spencer P.; and Evans, Mark S., "Dynamic Modeling of Compliant Constant-Force Compression Mechanisms" (2003). Faculty Publications. 465.

https://scholarsarchive.byu.edu/facpub/465 


\section{Dynamic Modeling of Compliant Constant-Force Compression Mechanisms}

\author{
Cameron Boyle \\ Guidance, Navigation \& Control \\ Raytheon \\ Tucson, AZ 85747 \\ Cameron_L_Boyle@raytheon.com
}

\author{
Larry L. Howell \\ Mechanical Engineering \\ Brigham Young University \\ Provo, Utah, 84602 \\ lhowell@et.byu.edu
}

\author{
Spencer P. Magleby \\ Mechanical Engineering \\ Brigham Young University \\ Provo, Utah, 84602 \\ magleby@byu.edu
}

\author{
Mark S. Evans \\ President, Direct Controls, Inc. \\ Direct Controls, Inc. \\ Provo, UT 84604 \\ evans@directcontrols.net
}

\section{Abstract}

A mathematical dynamic model is derived for compliant, constant-force compression-mechanisms, based on the pseudo-rigid-body model simplification of the device. The compliant constant-force compression mechanism (CFCM) is a slider mechanism incorporating large-deflection beams, which outputs near-constant-force across the range of its designed deflection. The equation of motion is successfully calibrated with empirical data from five separate mechanisms, comprising two basic configurations of CFCMs. The dynamic equation is derived from a generalized pseudo-rigid-body model. This allows every configuration to be represented by the same model, so a separate treatment is not required for each configuration. An unexpected dynamic trait of the constant-force mechanism is discovered. There exists a range of input deflection frequencies for which the output force of the mechanism is nearer to constant-force than it is with static input deflections.

\section{Introduction}

A constant-force compression mechanism provides a constant-force output over a range of deflections. Recent research has led to the development of practical constant-force compression mechanisms using principles of compliant mechanisms. These compliant, constant-force compression mechanisms (CFCM) offer the possibility of a new type of spring element for a variety of applications, many of which will require that the engineer have an understanding of the dynamic operating conditions that will cause the CFCM performance to vary from its static performance. 
Because the elastic deflections are large enough to be in the nonlinear range, the dynamic analysis can be quite complicated. Ideally, a simple method for obtaining a viable closed-form dynamic model would be desirable. Because the constant-force mechanism was discovered through application of the pseudo-rigid-body model [1], it follows that the same model might be used to adequately describe its dynamics.

The primary objective of this paper is to model the dynamic behavior of compliant constant-force compression mechanisms, and secondly to further evaluate the validity of the pseudo-rigid-body model as a dynamic modeling tool.

The pseudo-rigid-body model (PRBM) approximates the nonlinear deflection and force characteristics of a compliant mechanism's flexible members by assigning them appropriate torsional spring and rigid-link counterparts. The power of the PRBM is its ability to convert a difficult-to-analyze compliant mechanism into a familiar rigid-body mechanism which can be analyzed using traditional kinematic approaches. Though the PRBM has been shown to be valid for the static analysis and design of compliant mechanisms [2] [3] [4], very little research has been performed to explore its usefulness in dynamic analyses. If the model can be shown to accurately approximate the dynamic response of compliant mechanisms, then its usefulness will be extended further. The nature of the PRBM will not allow the static model to capture the higher eigenmodes for high driving frequencies. However, the ability to model compliant mechanisms in frequency ranges of interest using kinematic models (from the PRBM), provides valuable insight into the behavior of the devices that is not available from finite element models. This insight is critical for analysis and making engineering decisions for improving device performance.

The paper first presents a brief background on some key topics, and reviews literature related to the dynamic modeling of mechanisms. A dynamic model for CFCMs is then developed. The model is then calibrated and validated from testing. Findings from the modeling and testing are discussed followed by conclusions from this research.

\section{Background}

Compliant mechanisms are mechanisms that obtain some or all of their motion through the deflection of flexible members. Historically, the most common method of designing compliant mechanisms has been trial and error. However, the conception of the pseudo-rigid-body modeling technique and other approaches has successfully opened the way for simple design and analysis of many compliant mechanisms [2]. Topological optimization methods have also been developed to successfully design compliant mechanisms [5] [6] [7] [8]. 
Constant-force mechanisms are useful in applications requiring a constant force to be applied to a time-varying or non-uniform surface, such as grinding, swiping, deburing, welding, and assembly [9]. They may also be used to maintain constant force between electrical connectors [10] regardless of part variations; as gripping devices to hold delicate parts of varying size; for wear testing, where a constant force must be applied to a surface even as the surface is worn; in manufacturing processes that involve tool diameter changes such as grinding or honing; or as safety valves to maintain a system at constant pressure when power is lost. In these and other applications, the constant-force mechanism eliminates the need for more complex force control, replacing it with a simple mechanical device.

Compliant constant-force mechanisms are essentially compliant slider mechanisms with flexible and rigid segment dimensions optimized to minimize the variation in the output force over a designed range of displacement. A typical configuration of a mechanism is depicted in Figure 1. Given an input displacement $\Delta x$, this mechanism will yield the same force $F$ over the full range of its designed deflection, plus or minus a small variation.

Twenty-eight configurations of the compliant constant-force mechanism have been identified by type synthesis techniques [11]. The mechanism configurations studied in this research were selected because of their large range of motion and manufacturability.

The PRBM has been used almost exclusively for design and modeling of products where the elastic deflections are in the nonlinear range, but dynamic effects are not a large factor in mechanism performance. The fact that the PRBM has a proven track record as a reliable predictor of static behavior for compliant mechanisms [3] [4] begs the question of how well it would account for dynamic effects. This paper is a step in addressing this question by investigating the dynamic behavior of a particular type of compliant mechanism that could be used in dynamic loading situations.

Along with the emergence of the pseudo-rigid-body modeling technique in recent years, other methods for designing and/or analyzing large-deflection compliant mechanisms have arisen, including both static and dynamic methods. One approach is the formulation of an exact mathematical model to describe large-deflection beam mechanisms. Bisshopp and Drucker [12], Burns and Crossley [13], and Sevak and McLarnan [14] used elliptic integral solutions to derive ordinary nonlinear differential equations to represent cantilever beam mechanisms. Simo and Posbergh [15] performed a geometrically exact formulation of a three-dimensional compliant rod coupled with a rigid body, with no restriction on the degree of flexibility. Wang [16] set up a dynamic equation to describe the dynamics of an elastic four-bar linkage mechanism with large 


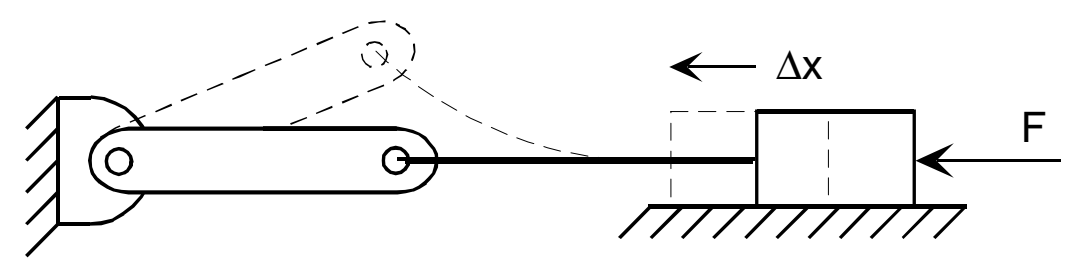

Figure 1. Compliant constant-force mechanism, configuration Class 1A-d.

elastic deformation. Atanackovic and Cveticanin [17] developed coupled partial differential equations in two coordinate systems to model the dynamic response of an unloaded pinned-roller beam under compression. Recently, Panza [18] published a nonlinear integro-partial differential equation representing the large-deflection dynamics of a compliant beam mechanism, incorporating mass loading and friction/damping effects.

A much more common approach to modeling compliant mechanisms is applying nonlinear finite-element modeling techniques in one form or another. For dynamic analysis, many methods have been successful, from the use of finite rotation theory [19] [20] and extended bond graph formulation [21], to the use of flexible multibody dynamics methods [22]. Another approach to dynamic modeling is the work of Pascal and Gagarina [23], who discretized flexible components by a RayleighRitz procedure, and then numerically simulated the dynamic response using dynamical codes devoted to rigid multibody systems. Similar work was performed by Petroka and Chang [24], Darcovich [25], and Zakhariev [26].

Lyon et al. [27] used the PRBM in conjunction with Lagrange's method to develop linear ordinary differential equations that successfully described compliant parallel-guiding mechanisms. It is the only work to date that investigates the possibility of using the PRBM to predict the dynamic response of compliant mechanisms.

Jenuwine and Midha [28] developed an exact constant-force mechanism model incorporating linear springs and rigid links. The compliant constant-force slider mechanism configurations, inspired by this original rigid-body device, came about through topological synthesis [1]. Millar et al. [3] presented a detailed development of compliant constant-force mechanism theory, with static validation of three configurations.

\section{Dynamic Model}

This section presents the derivation of a closed-form dynamic model for a constant-force mechanism configuration listed as "Class 1A-d," in Howell, [2] and shown in Figure 1. The configuration modeled consists of a rigid link pinned to ground and to a long flexible segment rigidly connected to a slider. This configuration was chosen for its ease of 
manufacturability, and because it has been used in applications such as a robotic end-effector [9]. First, the compliant mechanism is modeled as a rigid-body mechanism with lumped compliance using the pseudo-rigid-body model. Converting the mechanism to its rigid-body counterpart greatly simplifies kinematic and dynamic analysis by allowing the use of rigidbody modeling techniques. Lagrange's method is then used to obtain an equation of motion for the mechanism. The constantforce mechanism has only one degree of freedom, so only one generalized coordinate and one instance of Lagrange's equation are required. The choice of including non-conservative forces in the generalized force is explained, as well as the assumptions made in the model.

\section{The Pseudo-Rigid-Body Model}

The PRBM for the constant-force spring configuration Class 1A-d, is shown in Figure 2. The mechanism is converted to its rigid-body counterpart by using the PRBM for a cantilever beam with a force at the free end, as described below.

The flexible segment of length $l$ is replaced by two rigid links, links 3 and 6 , with lengths $r_{3}$ and $r_{6}$. Link 3's length is determined by the relation for the pseudo-rigid-body link's characteristic radius,

$$
r_{3}=\gamma l
$$

where $\gamma$ is the characteristic radius factor. The length of link 6 is then

$$
r_{6}=l-r_{3}
$$

The compliance of the flexible segment is represented by a torsional spring at the new pin (called the characteristic pivot) joining links 3 and 6 . The torsional spring constant $k$ for a cantilever beam with a force at the free end is given by

$$
k=\gamma K_{\Theta} \frac{E I}{l}
$$

where $K_{\Theta}$ is the stiffness coefficient (a nondimensionalized torsional spring constant), $E$ is the modulus of elasticity of the flexible segment, and $I$ is the moment of inertia of the flexible segment.

The average values of $\gamma(\gamma=0.85)$ and $K_{\Theta}\left(K_{\Theta}=2.65\right)$ over a wide range of loading conditions are used. For a more accurate $k$ that changes with deflection of the flexible segment, $\gamma$ and $K_{\Theta}$ can be expressed as functions of end-load angle. 


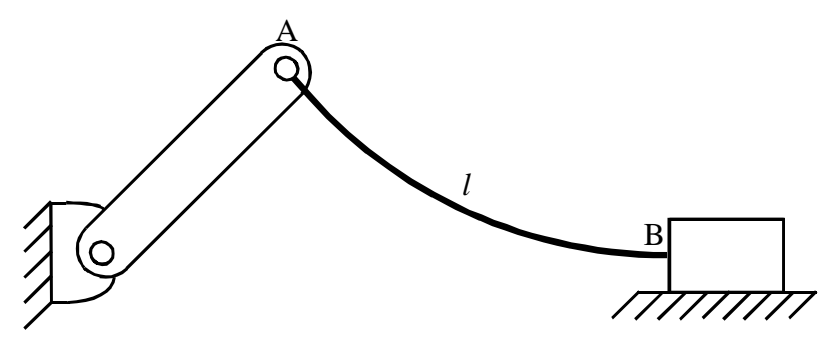

(a)

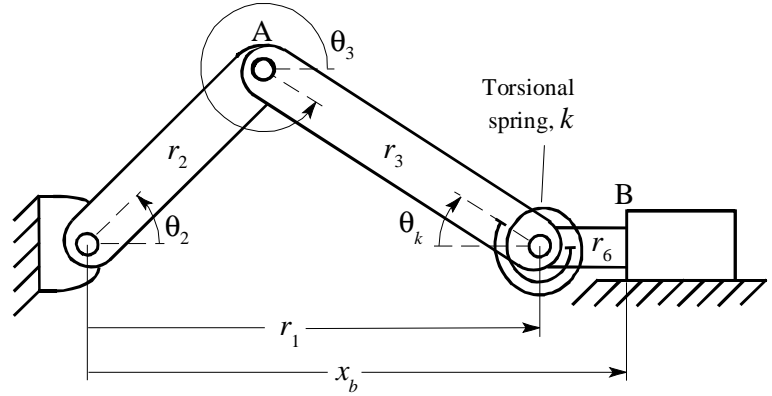

(b)

Figure 2. (a) Compliant constant-force mechanism, in its displaced state, and (b) its corresponding pseudo-rigid-body model.

\section{Formulating the Lagrangian}

As shown in Figure 3, $\theta_{2}(t)$ is selected as the generalized coordinate, corresponding to a generalized force $Q_{\theta_{2}}(t)$ to simplify the derivation. Note that because $\theta_{2}$ is an angle, its corresponding generalized force, $Q_{\theta}$, has units of moment.

The Lagrangian $\mathcal{L}$ is formed by taking the difference of the scalar quantities of kinetic energy $T$ and potential energy $V$ of the system,

$$
\mathscr{L}=T-V
$$

One way to formulate $T$ is to separate the motion of the mechanism inertias into both translation and rotation, as illustrated in Figure 4. The center of mass of each link translates along a predefined path as the mechanism moves, and each link rotates about its center of mass. The mass of link 6 can be lumped together with the mass of the slider since both travel along a linear path and neither rotates:

$$
m_{s}=m_{6}+m_{\text {slider }}
$$

The first three terms of the kinetic energy expression represent the translational energy of the system, and the last two represent the rotational energy:

$$
T=\frac{1}{2} m_{2} v_{c 2}^{2}+\frac{1}{2} m_{3} v_{c 3}^{2}+\frac{1}{2} m_{s} \dot{r}_{1}^{2}+\frac{1}{2} J_{c 2} \dot{\theta}_{2}^{2}+\frac{1}{2} J_{c 3} \dot{\theta}_{3}^{2}
$$

where $m_{i}$ is the mass, $v_{c i}$ is the velocity of the center of mass, $J_{c i}$ is the mass moment of inertia, and $\dot{\theta}_{i}$ is the angular velocity of links 2 and $3 ; \dot{r}_{1}$ is the velocity of the slider. The mass moments of inertia of links 2 and 3 are 


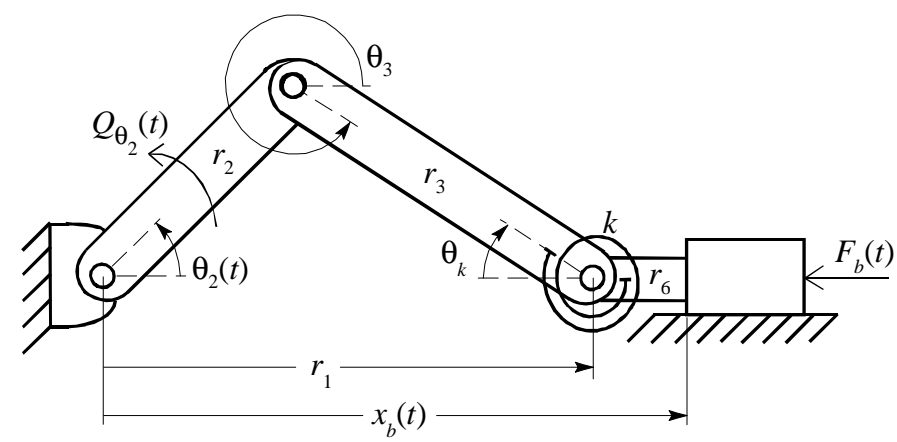

Figure 3. Reaction force $F_{b}(t)$ at the slider (output port); independent generalized coordinate $\theta_{2}(t)$ and generalized force $Q_{\theta_{2}}(t)$.

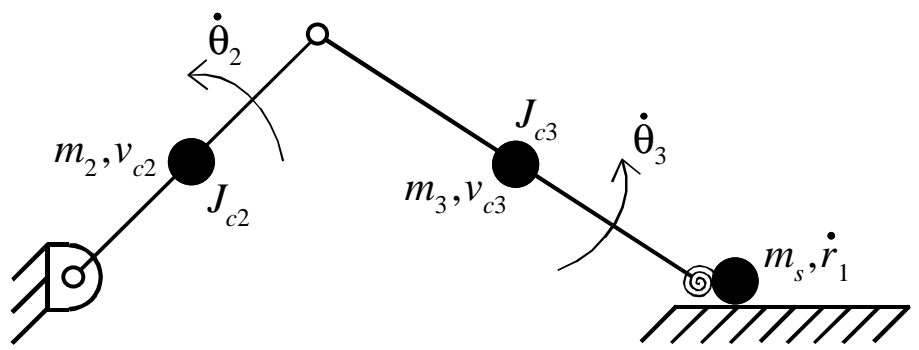

Figure 4. Translational and rotational motion of the mechanism links.

$$
J_{c i}=\frac{1}{12} m_{i} r_{i}^{2}
$$

Assuming the mechanism lies in a plane perpendicular to gravity, the potential energy of the system is simply the torsional spring energy

$$
V=\frac{1}{2} k \theta_{k}^{2}
$$

The Lagrangian $\mathscr{L}=T-V$ must be expressed in terms of the generalized coordinate $\theta_{2}$ and its time derivative $\dot{\theta}_{2}$ before forming Lagrange's equation. The following equations recast the variables in $T$ and $V$ in terms of $\theta_{2}$ and $\dot{\theta}_{2}$ :

$$
\begin{gathered}
v_{c 2}{ }^{2}=\frac{1}{4} r_{2}{ }^{2} \dot{\theta}_{2}^{2} \\
v_{c 3}{ }^{2}=r_{2}{ }^{2} \dot{\theta}_{2}^{2}+\frac{1}{4} r_{3}{ }^{2} \dot{\theta}_{3}{ }^{2}+r_{2} r_{3} \cos \left(\theta_{2}-\theta_{3}\right) \dot{\theta}_{2} \dot{\theta}_{3} \\
\dot{r}_{1}^{2}=\left[r_{2} \sin \theta_{2} \dot{\theta}_{2}+r_{3} \sin \theta_{3} \dot{\theta}_{3}\right]^{2} \\
\theta_{k}=\operatorname{asin}\left(\frac{r_{2}}{r_{3}} \sin \theta_{2}\right) \\
\theta_{3}=\operatorname{asin}\left(-\frac{r_{2}}{r_{3}} \sin \theta_{2}\right)
\end{gathered}
$$




$$
\dot{\theta}_{3}=-\frac{r_{2} \cos \theta_{2}}{\sqrt{r_{3}{ }^{2}-r_{2}{ }^{2} \sin ^{2} \theta_{2}}} \dot{\theta}_{2}
$$

It is also useful to express $x_{b}$ as a function of $\theta_{2}$ :

$$
x_{b}=r_{1}+r_{6}
$$

where

$$
r_{1}=r_{2} \cos \theta_{2}+\sqrt{r_{3}^{2}-r_{2}^{2} \sin ^{2} \theta_{2}}
$$

\section{Lagrange's Equation}

Using Lagrange's formulation, the equation of motion for the system is expressed as

$$
\frac{d}{d t}\left(\frac{\partial \mathscr{L}}{\partial \dot{\theta}_{2}}\right)-\frac{\partial \mathscr{L}}{\partial \theta_{2}}=Q_{\theta_{2}}
$$

When the derivatives of the Lagrangian are expanded out, the equation of motion for the system becomes

$$
\begin{aligned}
& \left(\left[m _ { 3 } \left(\frac{1}{2} \frac{r_{2}{ }^{5} \sin ^{3} \theta_{2} \cos ^{2} \theta_{2}}{\xi^{3 / 2}}+\frac{1}{3} \frac{r_{2}{ }^{4} r_{3}{ }^{2} \sin \theta_{2} \cos ^{3} \theta_{2}}{\xi^{2}}-\frac{1}{2} \frac{r_{2}{ }^{3} \sin ^{3} \theta_{2}}{\sqrt{\xi}}+\frac{r_{2}{ }^{3} \sin \theta_{2} \cos ^{2} \theta_{2}}{\sqrt{\xi}}\right.\right.\right. \\
& \left.-\frac{1}{3} \frac{r_{2}^{2} r_{3}^{2} \sin \theta_{2} \cos \theta_{2}}{\xi}+r_{2}^{2} \sin \theta_{2} \cos \theta_{2}\right) \\
& +m_{s}\left(\frac{r_{2}{ }^{6} \sin ^{3} \theta_{2} \cos ^{3} \theta_{2}}{\xi^{2}}+\frac{r_{2}^{5} \sin ^{3} \theta_{2} \cos ^{2} \theta_{2}}{\xi^{3 / 2}}-\frac{r_{2}{ }^{4} \sin ^{3} \theta_{2} \cos \theta_{2}}{\xi}+\frac{r_{2}{ }^{4} \sin \theta_{2} \cos ^{3} \theta_{2}}{\xi}\right. \\
& \left.\left.+2 \frac{r_{2}^{3} \sin \theta_{2} \cos ^{2} \theta_{2}}{\sqrt{\xi}}-\frac{r_{2}^{3} \sin ^{3} \theta_{2}}{\sqrt{\xi}}+r_{2}{ }^{2} \sin \theta_{2} \cos \theta_{2}\right)\right] \dot{\theta}_{2}^{2}+\left[m_{2}\left(\frac{1}{3} r_{2}^{2}\right)\right. \\
& +m_{3}\left(\frac{r_{2}^{3} \sin ^{2} \theta_{2} \cos \theta_{2}}{\sqrt{\xi}}+\frac{1}{3} \frac{r_{2}^{2} r_{3}^{2} \cos ^{2} \theta_{2}}{\xi}+r_{2}^{2}-r_{2}^{2} \cos ^{2} \theta_{2}\right) \\
& \left.\left.+m_{s}\left(\frac{r_{2}{ }^{4} \sin ^{2} \theta_{2} \cos ^{2} \theta_{2}}{\xi}+2 \frac{r_{2}^{3} \sin ^{2} \theta_{2} \cos \theta_{2}}{\sqrt{\xi}}+r_{2}^{2} \sin ^{2} \theta_{2}\right)\right] \ddot{\theta_{2}}\right)+\frac{k \operatorname{asin}\left(\frac{r_{2}}{r_{3}} \sin \theta_{2}\right) r_{2} \cos \theta_{2}}{\sqrt{\xi}}=Q_{\theta_{2}}
\end{aligned}
$$

where 


$$
\xi=r_{3}^{2}-r_{2}^{2} \sin ^{2} \theta_{2}
$$

The generalized force $Q_{\theta_{2}}$ consists of a moment due directly to the force $F_{b}$ acting on the slider $\tau_{F_{b}}$, a torque due to Coulomb pin friction $\tau_{C}$, and a term to compensate for unmodeled torque in the mechanism $\tau_{u m}$ :

$$
Q_{\theta_{2}}=\tau_{F_{b}}+\tau_{C}+\tau_{u m}
$$

The addition of the static terms $\tau_{C}$ and $\tau_{u m}$ to the dynamic model is discussed below.

Though more elaborate expressions for the Coulomb friction term $\tau_{C}$ are possible, the following simple relation provides a good approximation:

$$
\tau_{C}=C \theta_{2} \operatorname{sgn}\left(\dot{\theta}_{2}\right)
$$

Multiplying by $\theta_{2}$ is based on the idea that the classical Coulomb friction coefficient is likely to be proportional to the angle $\theta_{2}$, because the reaction force at the joint increases as $\theta_{2}$ increases.

The values of the Coulomb friction coefficient $C$ and the unmodeled torque $\tau_{u m}$ are chosen using experimental data from static tests, as described later.

Finally, to transform from the torque $\tau_{F_{b}}$ to the mechanism's output force $F_{b}$, we use the expression

$$
F_{b}=\tau_{F_{b}} / \frac{\partial x_{b}}{\partial \theta_{2}}
$$

where

$$
\frac{\partial x_{b}}{\partial \theta_{2}}=-r_{2} \sin \theta_{2}-\frac{r_{2}{ }^{2} \sin \theta_{2} \cos \theta_{2}}{\sqrt{\xi}}
$$

\section{Determining Coulomb Friction and Unmodeled Torque}

The Coulomb friction coefficient $C$ and the unmodeled torque $\tau_{u m}$ are chosen using empirical data from a static test [29] of a constant-force mechanism as shown in Figure 5. The modeled force with $\tau_{C}=\tau_{u m}=0$ is calculated directly from the input position $x_{b}$, a gradual ramp first in compression then expansion. The dynamic model equations (18) - (23) are used to calculate the predicted force. 
Clearly, without consideration for the static terms $\tau_{C}$ and $\tau_{u m}$, the modeled force does not correlate well with the measured force. To correct this, values for $C$ and $\tau_{u m}$ which draw the predicted force into better agreement with the measured force can be chosen empirically by performing a least-squares fit. Figure 6 illustrates this, with $C=0.045 \mathrm{~N} \cdot \mathrm{m}$ $\left(0.40 \mathrm{in} \cdot l b_{f}\right)$ and $\tau_{u m}=-0.228 \mathrm{~N} \cdot \mathrm{m}\left(-2.01 \mathrm{in} \cdot l b_{f}\right)$, resulting in a nice match between modeled and measured force.

The underlying assumption made by including Coulomb pin friction $\tau_{C}$ and unmodeled torque $\tau_{u m}$ terms in the model is that most of the mechanism's difficult-to-model characteristics can be represented by one or the other. Note that both $\tau_{C}$ and $\tau_{u m}$ do not relate to the dynamic terms of the model directly - they are static terms whose effects have already been observed in previous work [9].

The physical mechanism built to validate the model possesses these properties in the pin joints and the links. Associated with the pin joints are Coulomb friction in the pins, possible binding of the pins due to misalignment, unmodeled tolerances in the pin joints, and the effect of heating of the pins as they rotate. Associated with the mechanism's links is possible flexing of the rigid link (it's only twice as thick as the flexible segment), and the fact that the compliant segment is significantly stiffer where the hinge rivets to it. Friction and perhaps binding are taken care of by the $\tau_{C}$ term; the other effects

are taken up by the $\tau_{u m}$ term. These static terms are not unique to the PRBM, but similar terms are needed in static finite element analysis to account for these effects.

\section{Experimental Setup}

With the dynamic model developed and static terms determined, the model can now be validated through testing.

\section{Constant-Force Test Mechanism}

Figure 7 shows a picture of the constant-force mechanism used to validate the dynamic model derived earlier. It consists of a pair of configuration Class 1A-d constant-force mechanisms mounted to the same ground and sharing the same slider. Mounting two mechanisms opposite each other is useful because each cancels the moment induced by the other, and the issue of friction between slider and ground is eliminated.

To apply the dynamic model to the pair of constant-force springs, simply imagine the device split down its line of symmetry, as in Figure 8. The parameters of one of the halves (i.e. link lengths, masses, etc.) are used in the dynamic model 

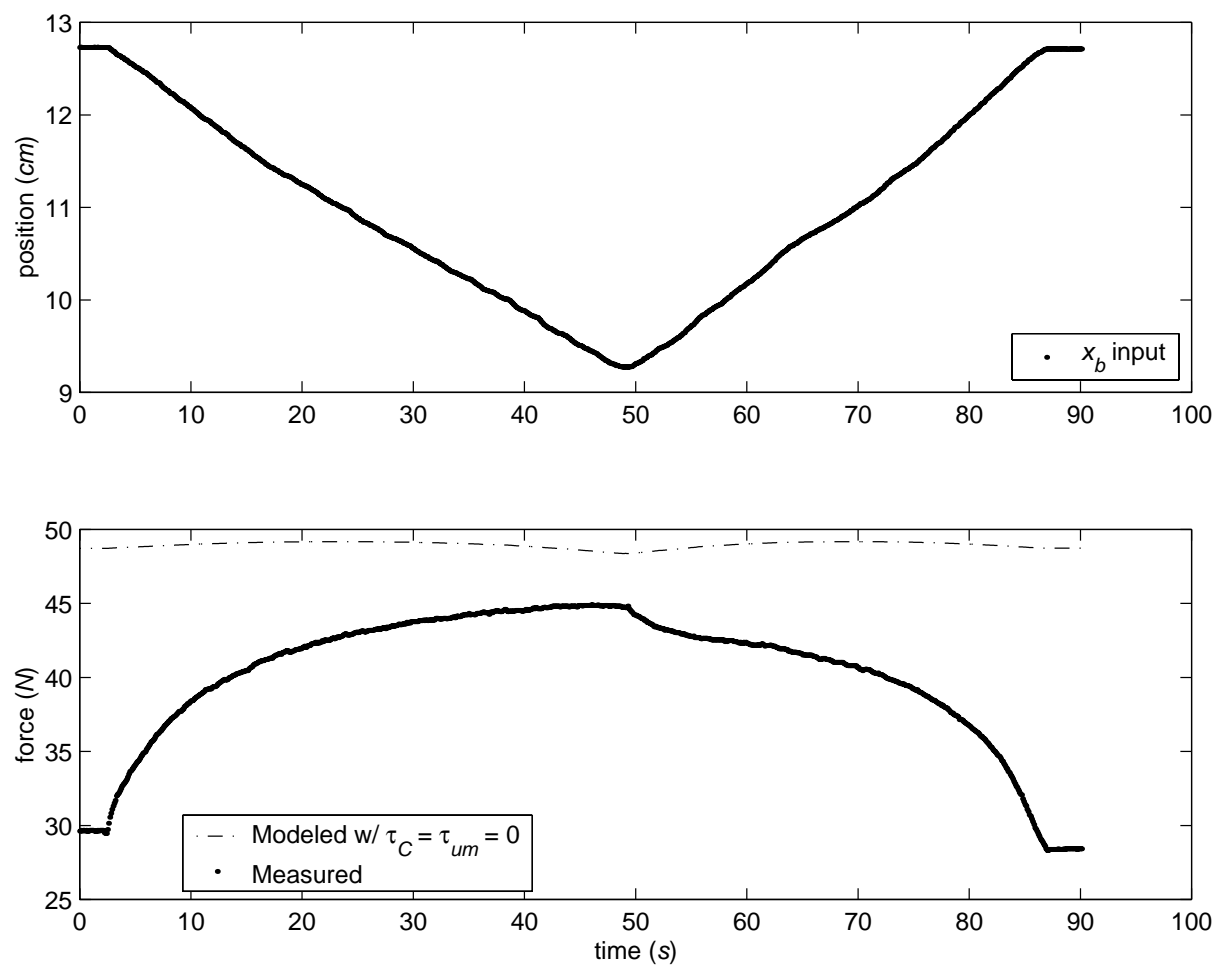

Figure 5. Modeled and measured force for static test input $x_{b}$; modeled force calculated with $\tau_{C}=\tau_{u m}=\mathbf{0}$.

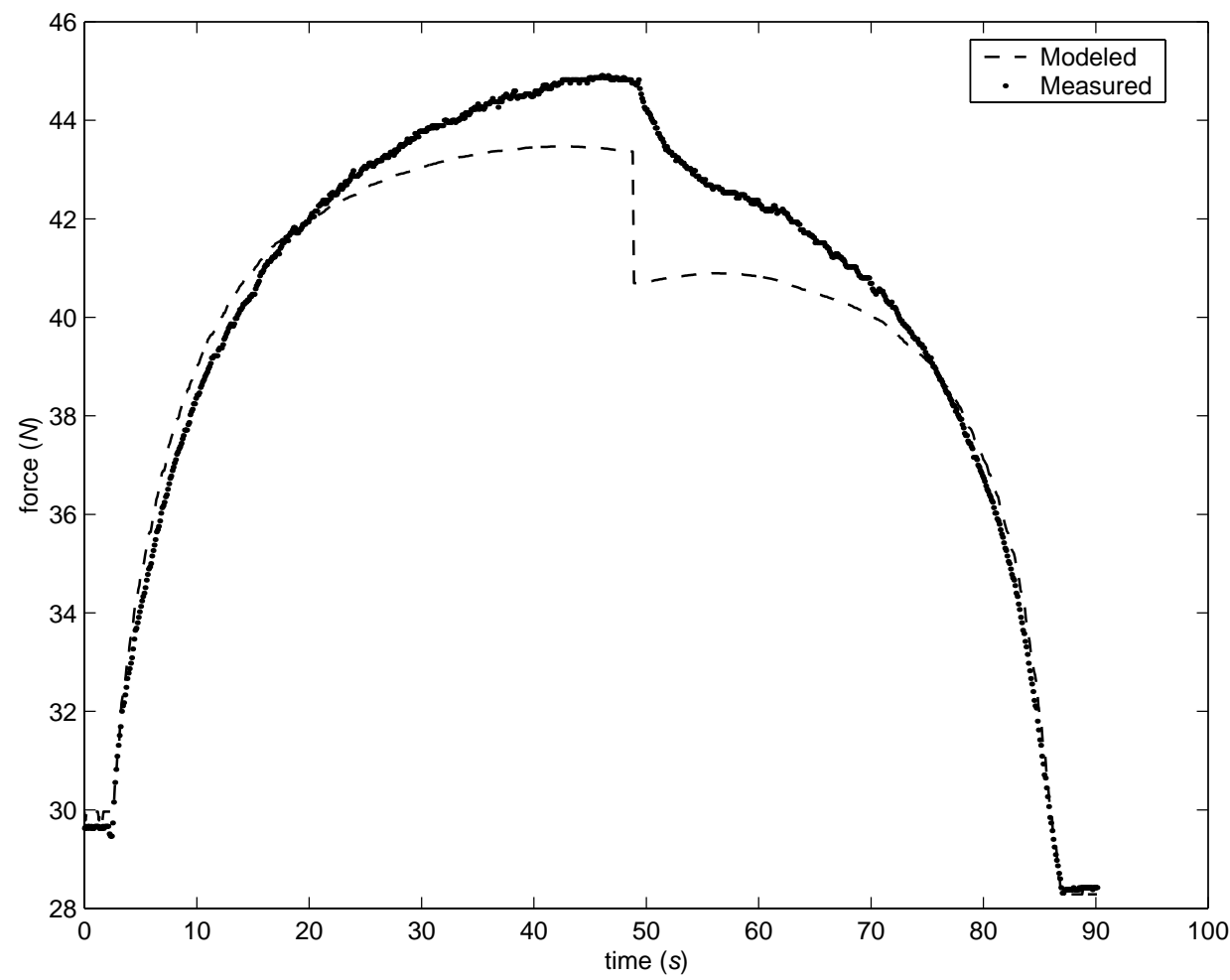

Figure 6. Modeled and measured force for static test input $x_{b}$; modeled force calculated with $C=0.045 N \cdot m$ and $\tau_{u m}=-0.228 N \cdot m$. 


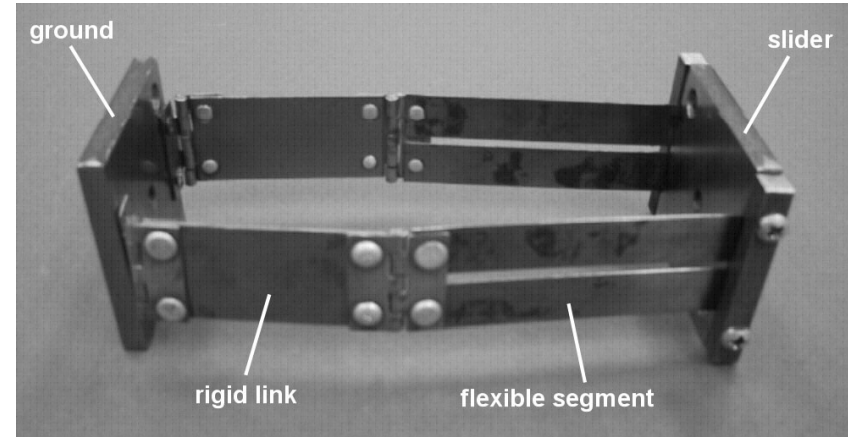

Figure 7. Constant-force mechanism for dynamic testing.

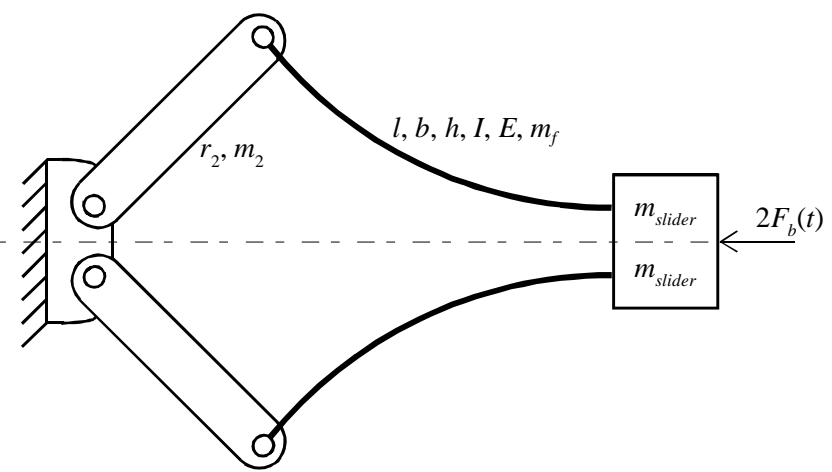

Figure 8. Constant-force test mechanism divided along its line of symmetry.

equations (19) - (24) to solve for the predicted force $F_{b}(t)$ of a single mechanism. The force expected at the output port of the constant-force spring pair is then twice $F_{b}(t)$. Note that the value of $m_{\text {slider }}$ is half the mass of the shared slider.

The relevant dimensions, masses (incorporating the masses of the hinges and clamps), and properties of the test mechanism are listed in Table 1 . The variables $b, h$, and $I$ are the width, thickness, and area moment of inertia of the flexible segment's cross section; $E$ and $m_{f}$ are the modulus of elasticity and mass of the flexible segment.

The test mechanism was designed to exhibit constant-force for a maximum deflection of $4.757 \mathrm{~cm}(1.873 \mathrm{in})$. The mechanism's total extended length is $13.007 \mathrm{~cm}(5.112 \mathrm{in})$.

The device's nominal constant-force $F_{\text {nom }}$ (doubled for the mechanism pair) is the magnitude of the output force for the idealized constant-force mechanism [2]. It can be computed as

$$
F_{n o m}=2 \frac{k}{r_{3}} \Phi=50.19 N \quad\left(11.29 l b_{f}\right)
$$

Where $\Phi$ is a nondimensionalized constant-force parameter that relates the link length and spring constant to the output force.

For this configuration $\Phi$ has a value of $\Phi=0.4773$ [3]. Differenct configurations have different values for $\Phi$.

\section{Experimental Setup}

Figure 9 shows a photograph and schematic of the experimental setup used to validate the constant-force mechanism dynamic model. The setup was designed to allow testing of the mechanism by cycling it at different frequencies. 


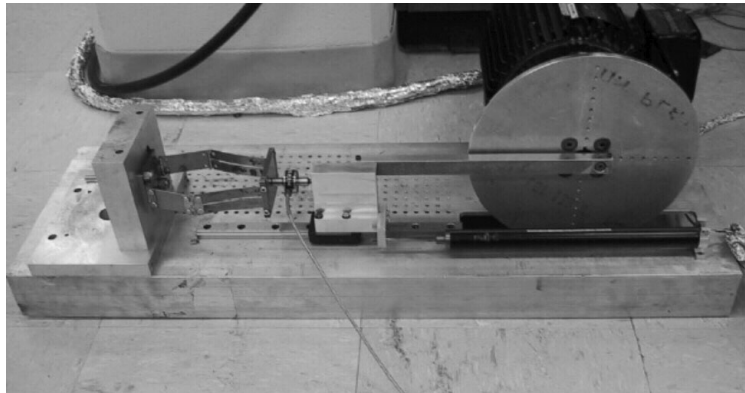

(a)

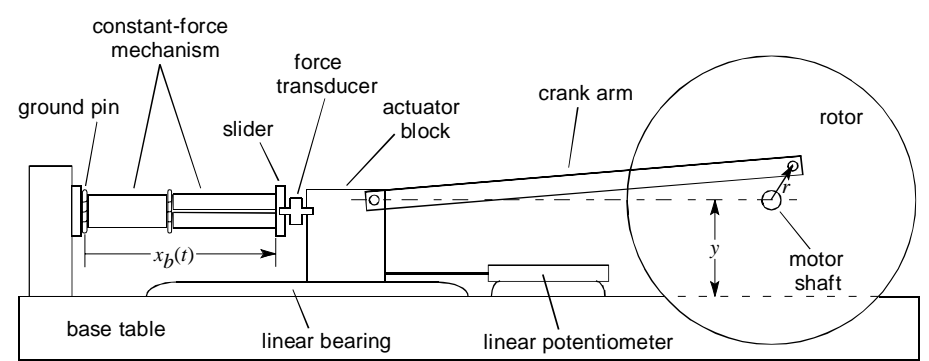

(b)

Figure 9. (a) Photograph of experimental setup for dynamic testing of constant-force mechanisms, and (b) schematic of experimental setup.

Table 1: Test mechanism dimensions, material properties, and masses (parameters used directly in dynamic model equations are emphasized).

\begin{tabular}{|c|c|}
\hline Parameter & Value \\
\hline$r_{2}$ & $5.490 \mathrm{~cm}$ \\
\hline$l$ & $7.517 \mathrm{~cm}$ \\
\hline$r_{3}=\gamma l$ & $6.390 \mathrm{~cm}$ \\
\hline$r_{6}=l-r_{3}$ & $1.128 \mathrm{~cm}$ \\
\hline$b$ & $2.540 \mathrm{~cm}$ \\
\hline$h$ & $0.064 \mathrm{~cm}$ \\
\hline$I=\frac{1}{12} b h^{3}$ & $5.420 \times 10^{-13} \mathrm{~m}^{4}$ \\
\hline$E$ & $206.8 \mathrm{GPa}$ \\
\hline$m_{2}$ & $13.8 \mathrm{~g}$ \\
\hline$m_{f}$ & $10.7 \mathrm{~g}$ \\
\hline$m_{3}=\gamma m_{f}$ & $9.1 \mathrm{~g}$ \\
\hline$m_{6}=m_{f}-m_{3}$ & $1.6 \mathrm{~g}$ \\
\hline$m_{\text {slider }}$ & $84.7 \mathrm{~g}$ \\
\hline$m_{s}=m_{\text {slider }}+m_{6}$ & $86.3 g$ \\
\hline$k$ & $3.359 N \cdot m$ \\
\hline$C$ & $0.055 \mathrm{~N} \cdot \mathrm{m}$ \\
\hline$\tau_{u m}$ & $-0.235 \mathrm{~N} \cdot \mathrm{m}$ \\
\hline
\end{tabular}


The constant-force test mechanism detailed in the proceeding section fastens to a thick aluminum ground, mounted perpendicular to a large aluminum table. In this manner both ground pins of the test mechanism are fixed with respect to the table. The test mechanism slider is actuated by a small aluminum block free to move across a linear bearing.

Bolted in-line between the actuator block and test mechanism, a load cell measures force exerted on the slider.

A linear potentiometer measures mechanism deflection. The potentiometer housing is mounted to the base table, with its positioning rod attached to the actuator block. Since the actuator block, load cell, and slider are bolted together, the potentiometer measures the position $x_{b}(t)$, a measurement of the point where the mechanism meets the slider, located with respect to where the mechanism attaches to ground. Measurement error of the position instrumentation was found to be $\pm 0.071 \mathrm{~cm}( \pm 0.028 \mathrm{in})$.

This setup allows for the testing of the constant-force mechanism prototype over a range of frequencies, up to about $85 \mathrm{rad} / \mathrm{s}(13.5 \mathrm{~Hz})$, above which there is danger of harming the setup equipment and/or the mechanism.

\section{Dynamic and Static Testing}

Dynamic tests were performed by setting the motor controller to run at a constant velocity, waiting for the system to reach steady-state, then collecting data at a sampling rate well above the input frequency. The position of the mechanism mount is adjusted so that at maximum expansion the mechanism has a slight preload.

\section{Empirical and Theoretical Findings}

Given a position input $x_{b}(t)$, and all physical parameters correctly defined, the dynamic model predicts the force expected at the constant-force mechanism's output port. A side-by-side comparison of modeled vs. measured force shows that the model predicts the dynamic response of the test mechanism quite well.

\section{Modeled vs. Measured Force}

Figures 10, 11 and 12 show position and force plots of three dynamic tests of increasing frequency, $\omega$. For each figure, the predicted force cycle is calculated directly from the input sinusoid cycle $x_{b}$ shown in the position plot, using the dynamic model equations (19) - (24). The measured data is banded by \pm 3 pooled sample standard deviations, $( \pm 3 \sigma)$, representing the $99.74 \%$ confidence interval of the measurement. 

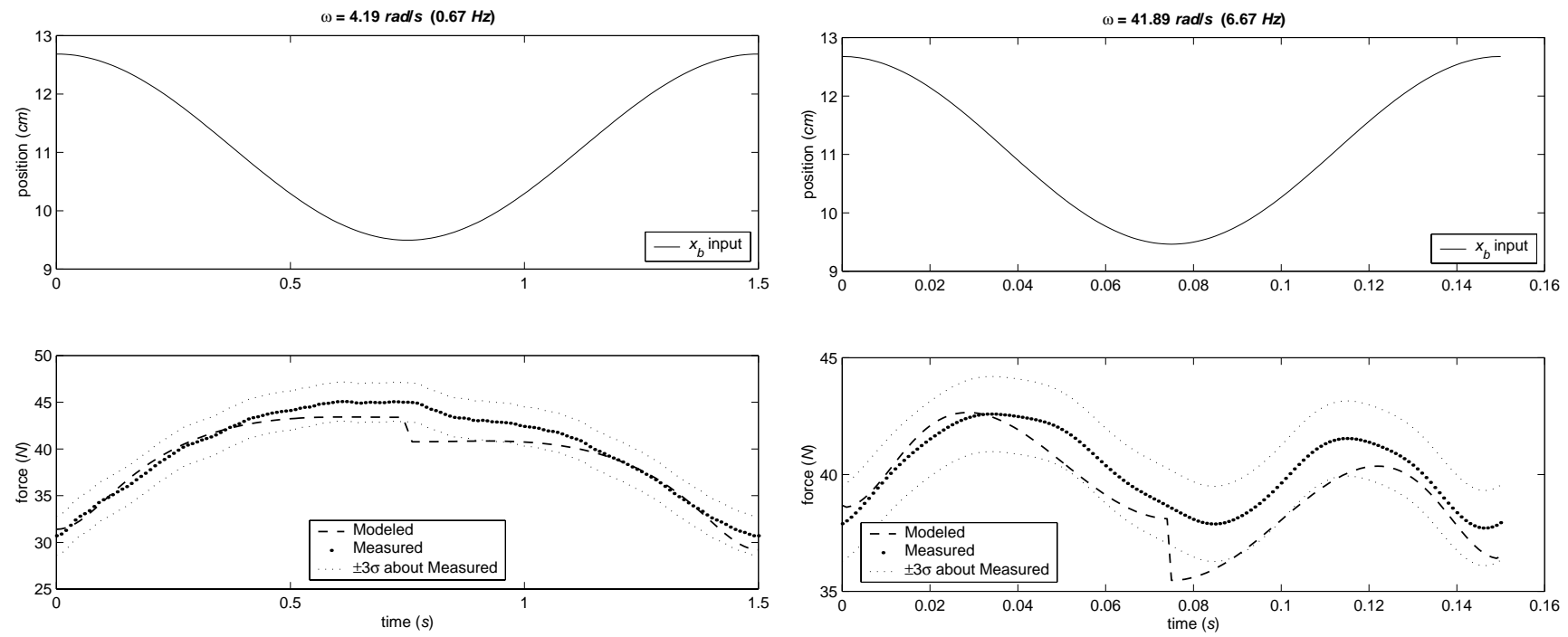

Figure 10. Predicted and measured force for

Figure 11. Predicted and measured force for sinusoidal input $x_{b}$ of $\omega=4 \mathrm{rad} / \mathrm{s}$.
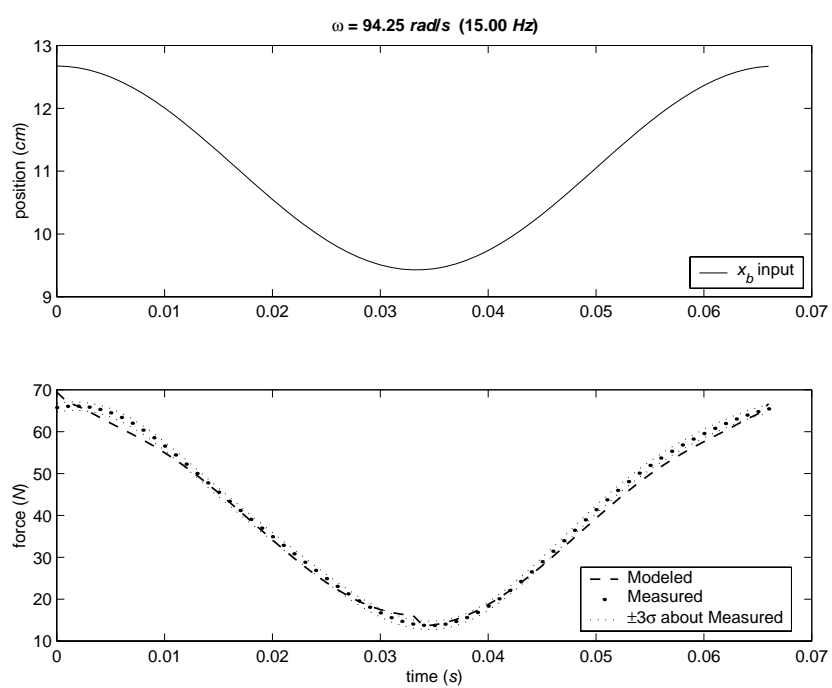

Figure 12. Predicted and measured force for sinusoidal input $x_{b}$ of $\omega=94 \mathrm{rad} / \mathrm{s}$.

While the modeled force does not match the measured force point for point, it does predict the average force, the peak-to-peak force difference, and the general shape of the force profile at any given frequency. These are important and useful elements to gather from a dynamic analysis of the system.

\section{Dynamic Characterization of the Model}

Although nonlinearities make it impossible to express an exact algebraic transfer function for the system, and no simple magnitude and phase plot can be shown, two useful plots can be analyzed. The median force and peak-to-peak force 
magnitude difference of the dynamic model as functions of frequency are shown in Figure 13. Each frequency assumes a sinusoidal position input with amplitude equal to the full $40 \%$ designed mechanism deflection (with a slight "predisplacement" to give a preload at full expansion). For good plot resolution, the dynamic model response is calculated at 400 separate and equally spaced frequencies.

The dynamic model represents the constant-force mechanism very well. Over the range of frequencies tested, the modeled force is within about $3 \%$ relative error of the measured force.

The heavy solid line represents the force predicted by the dynamic model with all parameters as defined in Table 1. The next two lines are purely theoretical and show what happens when the mass of the slider is set to zero, or when the mechanism has no inertia at all (all masses set to zero). Setting all the inertias to zero provides a baseline useful for comparison of the other curves, and setting the end mass to zero shows the dynamic response of the constant-force spring isolated as a separate "module". The fourth and fifth lines in the figure show the effect of multiplying the rigid link mass by four (which represents a possible improvement to the test mechanism, thickening the rigid link to ensure it doesn't flex), and the effect of reducing the end mass by $75 \%$. Lastly, the modeled force with Coulomb friction $\tau_{C}$ and unmodeled torque $\tau_{u m}$ set to zero is given.

Notice that each curve in the peak-to-peak force plot first decreases, then sustains a linear range before it starts to increase (all except for the third curve and last curve). This dip in magnitude difference is demonstrated nicely by Figures 10, 11 and 12. Clearly, the force profile of Figure 11 at $\omega=42 \mathrm{rad} / \mathrm{s}$ has a lower peak-to-peak force difference than the preceding and following figures at $\omega=4 \mathrm{rad} / \mathrm{s}$ and $\omega=94 \mathrm{rad} / \mathrm{s}$.

This very interesting and unexpected discovery from the peak-to-peak force plot is that there exists a range of frequencies over which a constant-force mechanism exhibits better constant-force behavior than for static loading. The device is designed to have nearly perfect constant force output under ideal static conditions. However, influences such as friction and elasticity of rigid components cause deviation from the constant-force behavior. The data shows that there are frequencies where the dynamic effects compensate for the nonideal characteristics of the mechanism. This range of frequencies coincides with the initial magnitude difference drop and most of the linear portion for each of the cases plotted in Figure 13. This unexpected finding significantly improves the likelihood that the compliant constant-force mechanism could be viable in 

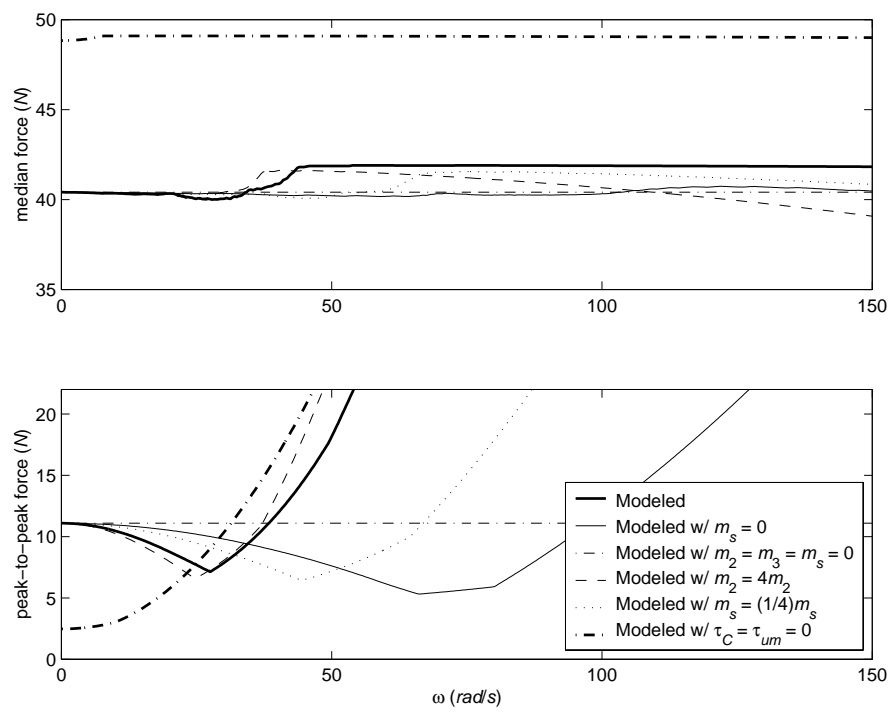

Figure 13. Frequency plots depicting the median force and peak-to-peak magnitude difference exhibited by the constant-force mechanism.

dynamic applications. For instance, if a designer were to use the test mechanism in an application and wanted to output as close to constant-force as possible, the mechanism would be designed at a frequency of $30 \mathrm{rad} / \mathrm{s}$ (see the heavy solid line of Figure 13). This would result in a constant-force mechanism with a median force of $40 N\left(9.0\left(b_{f}\right)\right.$ and a force variance of $\pm 3.5 N\left( \pm 0.79 l b_{f}\right)$, much better than the $\pm 6 N\left( \pm 1.35 l b_{f}\right)$ force variance the device demonstrates statically. Or if a designer wanted to maximize the range of frequencies over which the mechanism exhibits "better-than-static" constant-force, the slider mass could be minimized as much as possible. Figure 13 shows that the smaller the inertia of the slider, the higher the frequency before the force magnitude difference starts to rise.

Depending on what attributes are most desirable - a wide frequency band with moderately low peak-to-peak force, a single frequency with very low peak-to-peak force, or some other similar effect - the constant-force mechanism parameters can be optimized to achieve the desired results.

The peak-to-peak force and median force plots end at $150 \mathrm{rad} / \mathrm{s}$ for two reasons: (1) most everything of interest in the two plots occurs below this frequency, and (2) there is an upper limit (not necessarily $150 \mathrm{rad} / \mathrm{s}$ ) above which the constantforce mechanism starts to yield a negative force (i.e., will start to pull instead of push). This occurs when the force cycle exceeds a frequency where the peak-to-peak force equals twice the median force. For the test device (heavy solid line), this occurs at about $99 \mathrm{rad} / \mathrm{s}$. 


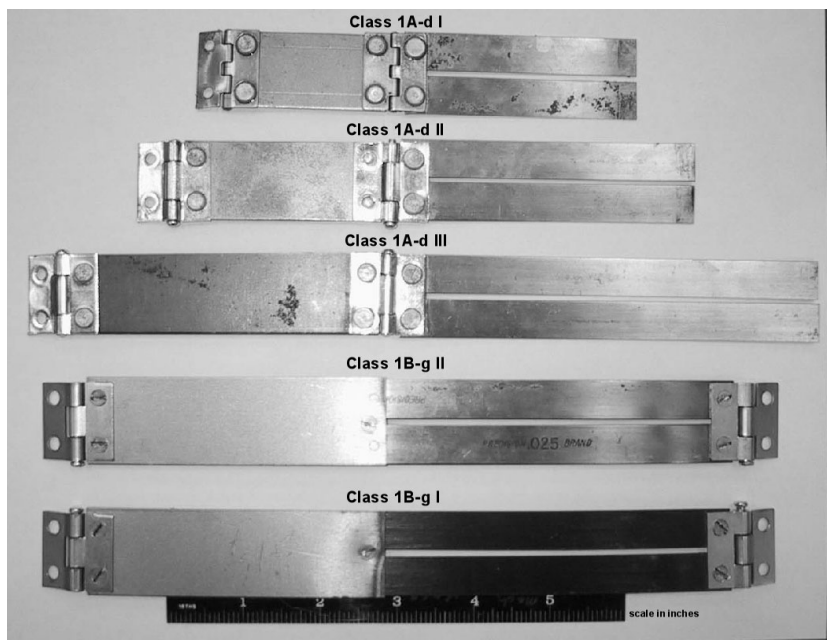

Figure 14. The five constant-force mechanisms tested.

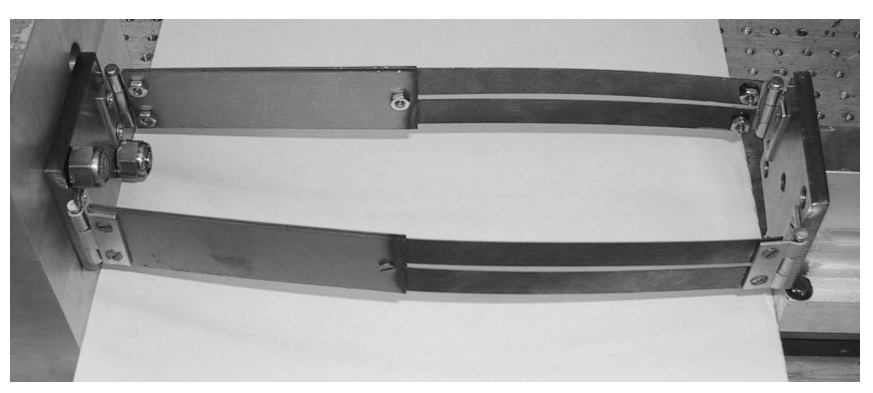

(a)

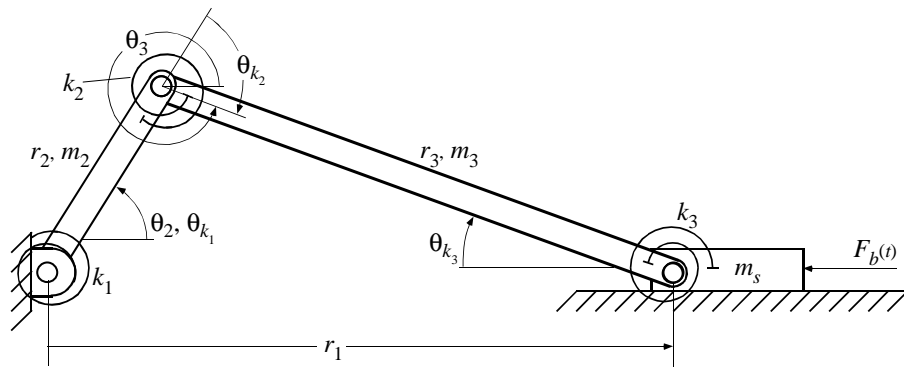

Figure 15. The generalized pseudo-rigid-body model for the compliant constant-force mechanism.

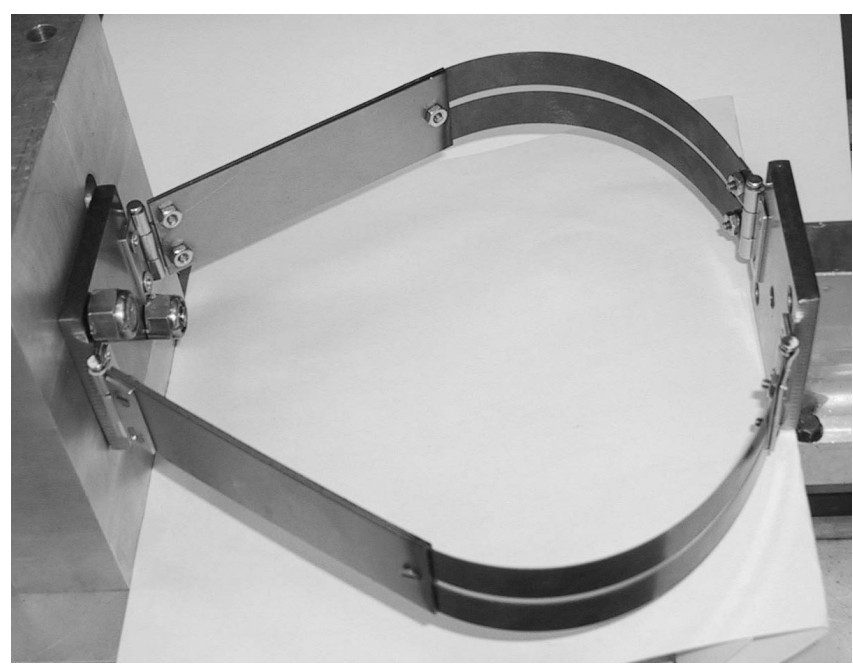

(b)

Figure 16. Mechanism Class 1B-g I, one of two Class 1B-g mechanisms tested; (a) fully expanded and (b) fully compressed.

In few applications will it be useful to give a constant-force mechanism a displacement input by attaching an actuator or surface directly to the slider; the two will usually be touching, but not rigidly connected. When the force becomes negative, this represents a situation where the slider breaks contact with the actuator or surface, possibly to cause an impact later. Of course these frequencies would be undesirable in most applications and should be avoided. 


\section{Testing of Additional Mechanisms}

Dynamic tests from four additional compliant constant-force mechanisms were evaluated. The four additional classes are shown in Figure 14. Two are of configuration Class 1A-d, the same configuration as the test mechanism described earlier, but with different dimensions. The latter two are of configuration Class 1B-g, whose PRBM is shown in Figure 15.

\section{Mechanism Description}

Figure 16 shows one of the Class 1B-g mechanisms, (a) fully expanded and (b) fully compressed (a deflection of $\left.\Delta x_{b}=0.40\left(r_{2}+r_{3}\right)\right)$. The mechanism parameters are listed in Table 2.

Every mechanism tested was designed to exhibit constant-force through a maximum deflection of $\Delta x_{b}=0.40\left(r_{2}+r_{3}\right)$. Table 3 lists each mechanism's extended length $x_{b \max }$ and fully compressed length $x_{b \min }$. It also gives each device's nominal constant-force $F_{\text {nom }}$ (doubled for the mechanism pairs), as [2]:

$$
F_{n o m}=2 \frac{k_{3}}{r_{3}} \Phi
$$

for the Class 1A mechanisms, and

$$
F_{\text {nom }}=2 \frac{k_{2}}{r_{3}} \Phi
$$

for the Class 1B mechanisms. The values for average nondimensionalized constant-force $\Phi$ for Class 1A and Class 1B constant-force mechanisms, are 0.4773 and 2.1513 , respectively [3].

\section{Test Results}

These four mechanisms showed trends similar to the first mechanism. All four of the mechanisms tested have a goodness of fit of modeled to measured force of $83 \%$ or better, and most were over $95 \%$.

Table 2: Extended and fully compressed mechanism lengths; nominal force for each mechanism.

\begin{tabular}{|l|c|c|c|c|}
\hline Parameter & 1A-d II & 1A-d III & 1B-g I & 1B-g II \\
\hline \hline$x_{b \max }$ & $16.711 \mathrm{~cm}$ & $24.933 \mathrm{~cm}$ & $22.029 \mathrm{~cm}$ & $22.029 \mathrm{~cm}$ \\
\hline$x_{b \min }$ & $10.598 \mathrm{~cm}$ & $15.813 \mathrm{~cm}$ & $13.218 \mathrm{~cm}$ & $13.218 \mathrm{~cm}$ \\
\hline$F_{n o m}$ & $31.19 \mathrm{~N}$ & $14.01 \mathrm{~N}$ & $19.47 \mathrm{~N}$ & $90.15 \mathrm{~N}$ \\
\hline
\end{tabular}


The higher relative error for these classes between measured and modeled force than mechanism Class 1A-d I is likely due to considerably large deflection in the pin joints. Test results show that all four mechanisms exhibit a range of frequencies over which the mechanism exhibits better constant-force behavior than at static conditions.

\section{Conclusions}

Five different mechanisms comprising two configurations of the compliant constant-force mechanism show good agreement between the dynamic model and measured results. This demonstrates that the pseudo-rigid-body model can be used to obtain a viable dynamic model for the compliant constant-force mechanism. In light of the simplicity the PRBM affords, this is a beneficial discovery, and the findings of this work encourage further investigation of using the PRBM for compliant mechanism dynamic analysis.

It was anticipated that the PRBM would approximate mechanism dynamics well, because the model's transformation from compliant mechanism to rigid-link mechanism does not result in a significant redistribution of mass. The PRBM lumps a compliant mechanism's distributed compliance at distinct points, but the changes it makes to the mechanism's distribution of

Table 3: Mechanism parameters (parameters used directly in dynamic model are emphasized).

\begin{tabular}{|l|c|c|c|c|}
\hline Parameter & 1A-d II & 1A-d III & 1B-g I & 1B-g II \\
\hline$r_{2}$ & $7.176 \mathrm{~cm}$ & $10.706 \mathrm{~cm}$ & $11.908 \mathrm{~cm}$ & $11.908 \mathrm{~cm}$ \\
\hline$r_{3}$ & $8.105 \mathrm{~cm}$ & $12.093 \mathrm{~cm}$ & $10.121 \mathrm{~cm}$ & $10.121 \mathrm{~cm}$ \\
\hline$r_{6}$ & $1.430 \mathrm{~cm}$ & $2.134 \mathrm{~cm}$ & & $2.540 \mathrm{~cm}$ \\
\hline$b$ & $2.540 \mathrm{~cm}$ & $2.540 \mathrm{~cm}$ & $2.540 \mathrm{~cm}$ & $0.064 \mathrm{~cm}$ \\
\hline$h$ & $0.064 \mathrm{~cm}$ & $0.064 \mathrm{~cm}$ & $0.038 \mathrm{~cm}$ & $5.420 \times 10^{-13} \mathrm{~m}$ \\
\hline$I$ & $5.420 \times 10^{-13} \mathrm{~m}^{4}$ & $5.420 \times 10^{-13} \mathrm{~m}^{4}$ & $1.171 \times 10^{-13} \mathrm{~m}^{4}$ & $206.8 \mathrm{GPa}$ \\
\hline$E$ & $206.8 \mathrm{GPa}$ & $206.8 \mathrm{GPa}$ & $206.8 \mathrm{GPa}$ & $50.1 \mathrm{~g}$ \\
\hline$m_{2}$ & $24.4 \mathrm{~g}$ & $35.8 \mathrm{~g}$ & $45.0 \mathrm{~g}$ & $14.3 \mathrm{~g}$ \\
\hline$m_{3}$ & $11.7 \mathrm{~g}$ & $16.8 \mathrm{~g}$ & $9.2 \mathrm{~g}$ & $85.5 \mathrm{~g}$ \\
\hline$m_{s}$ & $86.5 \mathrm{~g}$ & $87.0 \mathrm{~g}$ & $85.5 \mathrm{~g}$ & $-1.018 \mathrm{~N} \cdot \mathrm{m}$ \\
\hline$k_{3}$ & $2.648 \mathrm{~N} \cdot \mathrm{m}$ & $1.775 \mathrm{~N} \cdot \mathrm{m}$ & $0.4581 \mathrm{~N} \cdot \mathrm{m}$ & $2.121 \mathrm{~N} \cdot \mathrm{m}$ \\
\hline$C$ & $0.121 \mathrm{~N} \cdot \mathrm{m}$ & $0.029 \mathrm{~N} \cdot \mathrm{m}$ & $0.018 \mathrm{~N} \cdot \mathrm{m}$ & $0.144 \mathrm{~N} \cdot \mathrm{m}$ \\
\hline$\tau_{u m}$ & $-0.321 \mathrm{~N} \cdot \mathrm{m}$ & $-0.158 \mathrm{~N} \cdot \mathrm{m}$ & $-0.159 \mathrm{~N}$ & \\
\hline
\end{tabular}


mass are very small, so modeled and actual dynamic inertial forces should be reasonably consistent. Inertial forces acting on the mechanism masses, it is assumed, dominate over other dynamic forces.

The dynamic model derived for the constant-force compliant mechanism reveals an advantageous dynamic property exhibited by the device, which is also borne out by empirical data. The constant-force mechanism yields better constant-force behavior for certain driving frequencies than it does statically. The ability to manipulate this behavior would be useful to anyone designing compliant constant-force mechanisms. By changing mechanism parameters, a designer can optimize this feature to meet design specifications.

\section{Acknowledgements}

The authors gratefully acknowledge the support of the Utah Center of Excellence Program and the National Science Foundation through grant 9624574. Special thanks to Megan McAllister for work on the manuscript.

\section{References}

[1] Howell, L.L., Midha, A., and Murphy, M.D, 1994, "Dimensional Synthesis of Compliant Constant-Force Slider Mechanisms," Machine Elements and Machine Dynamics, DE, Vol. 71, pp. 509-515.

[2] Howell, L.L., 2001, Compliant Mechanisms, John Wiley \& Sons, New York.

[3] Millar, A.J., Howell, L.L., and Leonard, J.N., 1996, "Design and Evaluation of Compliant Constant-Force Mechanisms," Proceedings of the 1996 ASME Mechanisms Conference, 96-DETC/MECH-1209.

[4] Howell, L.L., Midha, A., and Norton, T.W., 1996, "Evaluation of Equivalent Spring Stiffness for Use in a Pseudo-RigidBody Model of Large-Deflection Compliant Mechanisms," ASME Journal of Mechanical Design, Vol. 118, pp.126-131.

[5] Frecker, M. Ananthasuresh, G.K., Nishiwaki, S., Kikuchi, N., and Kota, S., 1997, “Topological Synthesis of Compliant Mechanisms Using Multi-Criteria Optimization,” ASME Journal of Mechanical Design, Vol. 119, No. 2, pp. $238-245$.

[6] Saxena, A. and Ananthasuresh, G.K., 2001, "Topology Synthesis of Compliant Mechanisms for Nonlinear ForceDeflection and Curved Path Specifications," Journal of Mechanical Design, 123, pp. 33-42.

[7] Pedersen, C.B.W., Buhl, T., Sigmund, O., 2001, “Topology Synthesis of Large-Displacement Compliant Mechanisms,” International Journal for Numerical Methods in Engineering, 50, pp. 2683-2705.

[8] Ananthasuresh, G.K., and Kota, S., 1995, "Designing Compliant Mechanisms," Mechanical Engineering Magazine, Vol. 117 No. 11, November, pp. 96-96.

[9] Evans, M.S. and Howell, L.L., 1999, “Constant-Force End-Effector Mechanism," Proceedings of the IASTED International Conference, Robotics and Applications, Oct. 28-30, Santa Barbara, CA, USA, pp. 250-256.

[10] Weight, B., Mattson, C., Magleby, S.P., and Howell L.L., "Near-Constant-Force Electrical Contacts," submitted for publication in Journal of Electronics Packaging, Trans. ASME.

[11] Murphy, M.D., Midha, A., and Howell, L.L., "Methodology for the Design of Compliant Mechanisms Employing Type Synthesis Techniques with Example," Mechanism Synthesis and Analysis, DE-Vol. 70, 23rd ASME Biennial Mechanisms Conference, 1994, pp. 61-66.

[12] Bisshopp, K.E., and Drucker, D.C., 1945, "Large Deflection of Cantilever Beams," Quarterly of Applied Mathematics, Vol. 3, No. 3, pp. 272-275.

[13] Burns, R.H., and Crossley, F.R.E, 1968, “Kinetostatic Synthesis of Flexible Link Mechanisms,” ASME Paper No. 68- 
Mech-36.

[14] Sevak, N.M., and McLarnan, C.W., 1974, "Optimal Synthesis of Flexible Link Mechanisms with Large Static Deflections," ASME Paper No. 74-DET-83.

[15] Simo, J.C., and Posbergh, T.A., 1988, "Nonlinear Dynamics of Flexible Structures: Geometrically Exact Formulation and Stability," Proceeding of the 27th Conference on Decision and Control, IEEE FA2-12:15, pp. 1732-1737.

[16] Wang, Y., 1997, "Dynamics of an Elastic Four Bar Linkage Mechanism with Geometric Nonlinearities," Nonlinear Dynamics, Vol. 14, pp. 357-375.

[17] Atanackovic, T.M., and Cveticanin, L.J., 1996, "Dynamics of Plane Motion of an Elastic Rod," ASME Journal of Applied Mechanics, Vol. 63, pp. 392-398.

[18] Panza, M.J., 2000, "Mathematical Model for Large Deflection Dynamics of a Compliant Beam Mechanism," Proceedings of the 1997 ASME Design Engineering Technical Conferences, DETC2000/MECH-14146.

[19] Honke, K., Inoue, Y., Hirooka, E., and Sugano, N., 1997, “A Study on the Simulation of Flexible Link Mechanics Based on FEM," Proceedings of the 1997 ASME Design Engineering Technical Conferences, DETC97/VIB-4210.

[20] Sugano, N., Honke, K., Imanishi, E., and Hashimoto, K., 1999, "A Study on the Dynamic Analysis of Flexible and Rigid Link System," Proceedings of the 1997 ASME Design Engineering Technical Conferences, DETC99/VIB-8247.

[21] Yen, C. and Masada, G.Y., 1994, "Dynamic Analysis of Flexible Bodies Using Extended Bond Graphs," Journal of Dynamic Systems, Measurement, and Control, Vol. 116, No. 1, pp. 66-72.

[22] Megahed, S.M. and Hamza, K.T., 2000, "Modeling of Planar Flexible Link Manipulators: Parameter Identification Using Genetic Algorithm," Proceedings of the 2000 ASME Mechanisms Conference, DETC2000/MECH-14184.

[23] Pascal, M. and Gargarina, T., 1999, "Dynamical Simulation of Flexible Multibody System by Using a Rigid Body Model," Proceedings of DETC 99 ASME Design Engineering Technical Conferences, September 12-15, Las Vegas, Nevada, DETC99/VIB-8230.

[24] Petroka, R.P., and Chang, L., 1989, "Experimental Validation of a Dynamic Model (Equivalent Rigid Link System) on a Single-Link Flexible Manipulator," Journal of Dynamic Systems, Measurement, and Control, Vol. 111, pp. 667-672.

[25] Darcovich, J., Angeles, J., and Misra, A.K., 1992, "Dynamics of Single-Loop Mechanisms with Flexible Links," Flexible Mechanisms, Dynamics, and Analysis, DE, Vol. 47, pp. 453-460.

[26] Zakhariev, E.V., 1999, "Nonlinear Dynamics of Rigid and Flexible Multibody Systems," Proceedings of the 1999 ASME Design Engineering Technical Conferences, DETC99/VIB-8248.

[27] Lyon, S.M., Evans, M.S., Erickson, P.A., and Howell, L.L., "Prediction of the First Modal Frequency of Compliant Mechanisms Using the Pseudo-Rigid-Body Model," Journal of Mechanical Design, Trans. ASME, Vol. 121, No. 2, pp. 309313, 1999.

[28] Jenuwine, J.G., and Midha, A., 1989, "Design of an Exact Constant-Force Generating Mechanism," Proceedings of the 1st National Applied Mechanisms \& Robotics Conference, Vol. II, Cincinnati, Ohio, pp. 10B-4-1 - 10B-4-5.

[29] Boyle, C., 2001, A Closed-Form Dynamic Model of the Compliant Constant-Force Mechanism Using the Pseudo-RigidBody Model, MS Thesis, Brigham Young University

[30] Thomson, W.T., and Dahleh, M.D., 1998, Theory of Vibration with Applications, 5th edition, Prentice Hall, Upper Saddle River, New Jersey. 